\title{
Original Research \\ Uterine artery chemoembolization for management of unruptured interstitial pregnancy diagnosed in the early first trimester
}

\author{
Akihiro Takeda ${ }^{1, *}$, Shohei Iyoshi ${ }^{1}$, Satoshi Tamauchi ${ }^{1}$, Wataru Koike ${ }^{2}$ \\ ${ }^{1}$ Department of Obstetrics and Gynecology, Gifu Prefectural Tajimi Hospital, Tajimi, 507-8522 Gifu, Japan \\ ${ }^{2}$ Department of Diagnostic Radiology, Gifu Prefectural Tajimi Hospital, Tajimi, 507-8522 Gifu, Japan \\ *Correspondence: gyendoscopy@gmail.com (Akihiro Takeda) \\ Academic Editor: Antonio Simone Laganà \\ Submitted: 5 July 2021 Revised: 13 August 2021 Accepted: 18 August 2021 Published: 12 January 2022
}

\begin{abstract}
Background: With the increased frequency of diagnosis of interstitial pregnancy in the early first trimester, non-surgical management of unruptured interstitial pregnancy has become an important issue. However, management of unruptured interstitial pregnancy by uterine artery chemoembolization (UACE) with dactinomycin has never been evaluated via a case series. Methods: With this aim, a retrospective review of electronic chart records over a five-year period was performed, and a series of cases of unruptured interstitial pregnancy during the first trimester was extracted. Diagnostic procedures included ultrasonography, magnetic resonance imaging (MRI), and laparoscopic examination, if necessary. Conservative treatment regimen included UACE. Additional administration of methotrexate (MTX) was considered when an insufficient decline of serum $\beta$-hCG was noted. Clinical characteristics and treatment outcomes are described. Results: Among four women diagnosed with unruptured interstitial pregnancy at six weeks of gestation, one case was managed by laparoscopic cornuostomy due to concerns of rupture after the identification of thinning of the myometrium, whereas the other three cases were initially managed by UACE. One case of proximal interstitial pregnancy was diagnosed solely by MRI, whereas two cases of distal interstitial pregnancy was diagnosed by exploratory laparoscopy. Two cases were successfully managed by UACE alone, whereas one case required additional systemic administration of MTX to achieve resolution of gestational products after UACE. Thereafter, one patient conceived spontaneously and experienced a successful vaginal birth. Conclusions: This small case series emphasizes that UACE is a feasible minimally invasive option for the management of unruptured interstitial pregnancy identified in the early first trimester.
\end{abstract}

Keywords: Dactinomycin; Diagnostic imaging; Diagnostic laparoscopy; Interstitial pregnancy; Uterine artery chemoembolization

\section{Introduction}

Ectopic pregnancy occurs when the fertilized ovum implants outside the endometrial cavity [1]. The reported incidence of ectopic pregnancy occurs in $1.3 \%$ to $2 \%$ of all pregnancies [1]. Therefore, ectopic pregnancies represent an important risk factor for maternal morbidity and mortality in early pregnancy. Implantation of the fertilized ovum in the fallopian tube accounts for approximately $95 \%$ to $99 \%$ of ectopic pregnancies [1], whereas other localizations, such as ovarian, cervical, abdominal, and hepatic implants, contribute to rare occurrences ( $1 \%$ to $4 \%$ ) of ectopic pregnancy $[1,2]$.

Interstitial pregnancy is defined as a rare and potentially dangerous type of ectopic gestation involving implantation in the uterine interstitial portion of the fallopian tube $[3,4]$. Interstitial pregnancies account for only $2 \%$ to $4 \%$ of tubal pregnancies [3]. However, it has been reported that the mortality rate is as high as $2.5 \%$, which is seven times higher than that of typical ectopic pregnancies [4].

If rupture occurs in the interstitial portion of the uterus, which includes abundant blood supply from a complex pelvic vascular network composed of the uterine artery and anastomosing ovarian artery, definitive management involves life-saving intervention either by laparotomy or, more recently, laparoscopy [3]. However, for the management of hemodynamically stable cases without rupture, a wide range of surgical and non-surgical procedures are available [3-14].

With the recent development of diagnostic and therapeutic modalities, diagnosis of interstitial pregnancy in the early first trimester has become increasingly common [3]. Consequently, non-surgical management of unruptured interstitial pregnancy has become a prevalent issue [7,11-13].

Previously reports demonstrated that uterine artery chemoembolization (UACE), which involves uterine arterial chemotherapy infusion followed by endovascular embolization, is a feasible option for managing interstitial pregnancy [4-6].

In the present study, the therapeutic value of UACE for the management of unruptured interstitial pregnancy diagnosed in the early first trimester was retrospectively evaluated in a case series.

\section{Patients and methods}

\subsection{Patient selection}

This was a retrospective single-center study conducted at Gifu Prefectural Tajimi Hospital (Tajimi, Gifu, Japan). The inclusion period was from January 2014 to Decem- 
ber 2018. Initially, cases that met the International Classification of Diseases related to ectopic pregnancy (633.10, $633.11,633.20,633.80,633.81,633.90)$ were retrieved from the hospital electronic chart record. A manual check of all records was performed to identify cases of interstitial pregnancies. Among cases of interstitial pregnancies, only unruptured interstitial pregnancies diagnosed in the early first trimester were extracted, and cases with advanced gestation calculated from the last menstrual period at initial evaluation were excluded.

Detailed information about each patient, including demographics, gynecological and obstetrics history, and previous surgery, was retrieved from a review of clinical records and diagnostic images. Concerning the characteristics of interstitial pregnancies, dates of admission and discharge from the hospital, initial level of $\beta$-hCG, findings obtained by ultrasound and magnetic resonance imaging (MRI), presence of embryo and cardiac activity, mode of treatment, complications, post-treatment outcomes, and date of completion of treatment was examined. If the patient was treated surgically, then the surgical records were also evaluated.

Only patients that were observed for at least a year after the completion of treatment were included. Informed written consent was obtained from the patients regarding the use of their data for the study and publication of their disease status and clinical characteristics. Protective measures were taken to conceal the identity of patients. The Institutional Review Board of Gifu Prefectural Tajimi Hospital approved the current study (IRB number: 2020-OG3).

The datasets generated and analyzed during the current study are not publicly available, as they contain information that could compromise patient confidentiality. However, they are available from the corresponding author [A.T.] upon reasonable request.

\subsection{Diagnosis of unruptured interstitial pregnancy}

Women with suspected ectopic pregnancy were initially evaluated by transvaginal ultrasonography and measurements of the serum $\beta$-hCG level $[5,6]$. Typical presentation of interstitial pregnancy could be diagnosed by ultrasonography according to the established criteria for diagnosis: an empty uterine cavity, gestational sac located at least $1 \mathrm{~cm}$ from the lateral aspect of the uterus and lateral to the endometrial cavity, and a thin layer $(<5 \mathrm{~mm})$ of myometrium surrounding the gestational sac $[15,16]$.

When definitive diagnosis of interstitial pregnancy was difficult to determine but was suspected on initial ultrasonography, MRI was performed to locate the gestational sac. If necessary, three-dimensional computed tomographic angiography (3D-CTA) was performed to assess the degree of uteroplacental neovascularization [5,6]. After the images of each case were cooperatively evaluated by gynecologists and diagnostic radiologists, a consensus was made on the diagnosis and strategy for individual management.

Criteria for MRI diagnosis of interstitial pregnancy were a thickened endometrium without signs of gestation in the uterine cavity and identification of a gestational saclike structure within the muscular layer of the uterine cornus adjacent to the uninterrupted junctional zone $[5,6]$.

When the precise location of a gestational sac-like structure could not be determined even with the addition of MRI, exploratory single-port laparoscopy through an umbilical incision [17] was performed. If isthmic tubal pregnancy was confirmed, thus eliminating the possibility of interstitial pregnancy, laparoscopic excision of the affected fallopian tube was planned. If interstitial pregnancy was confirmed, either laparoscopic excision of gestational products was completed, or diagnostic laparoscopy followed by chemoembolization was performed based on the surgeon's decision while considering the patients' wish according to the informed consent. Depending on the site where gestational products were identified, interstitial pregnancy was further classified into proximal [6] or distal interstitial pregnancy [5].

\subsection{Uterine artery chemoembolization}

If immediate uterine devascularization with cytotoxic drugs was deemed necessary to remove interstitial gestational products, urgent digital subtraction angiography was performed. After a vascular mass derived from interstitial gestational products was confirmed, the branch of the uterine artery supplying the interstitial pregnancy was selectively catheterized under fluoroscopic guidance $[5,6]$.

UACE was performed by infusing dactinomycin $(0.5$ mg/body; Cosmegen; Merck \& Co., Inc., Whitehouse Station, NJ, USA) intra-arterially, followed by endovascular embolization using gelatin sponge particles (Serescue; Nippon Kayaku Co., Tokyo, Japan) as reversible embolization materials $[5,6]$.

After UACE, serial measurements of the serum $\beta$ hCG level were recorded every other day until $50 \%$ of the pre-treatment level was achieved, followed by weekly measurements until the serum $\beta$-hCG level decreased below the cut-off level $(<6 \mathrm{mIU} / \mathrm{mL})[5,6]$. Nonlinear disappearance of serum hCG when plotted on a semi-logarithmic graph was judged as a significant delay in the decline of the hCG value, which was managed by the administration of systemic methotrexate (MTX) $\left(50 \mathrm{mg} / \mathrm{m}^{2}\right)[5,6]$.

\section{Results}

\subsection{Study group classification of ectopic pregnancy}

Of 125 women with ectopic pregnancy, eight consecutive cases $(6.4 \%)$ were identified as interstitial pregnancies (Fig. 1). Gestational age was calculated based on the last documented menstrual period. Four cases with ruptured interstitial pregnancy, which were managed by laparoscopic surgery, were excluded from the current study.

Unruptured interstitial pregnancy was diagnosed in 


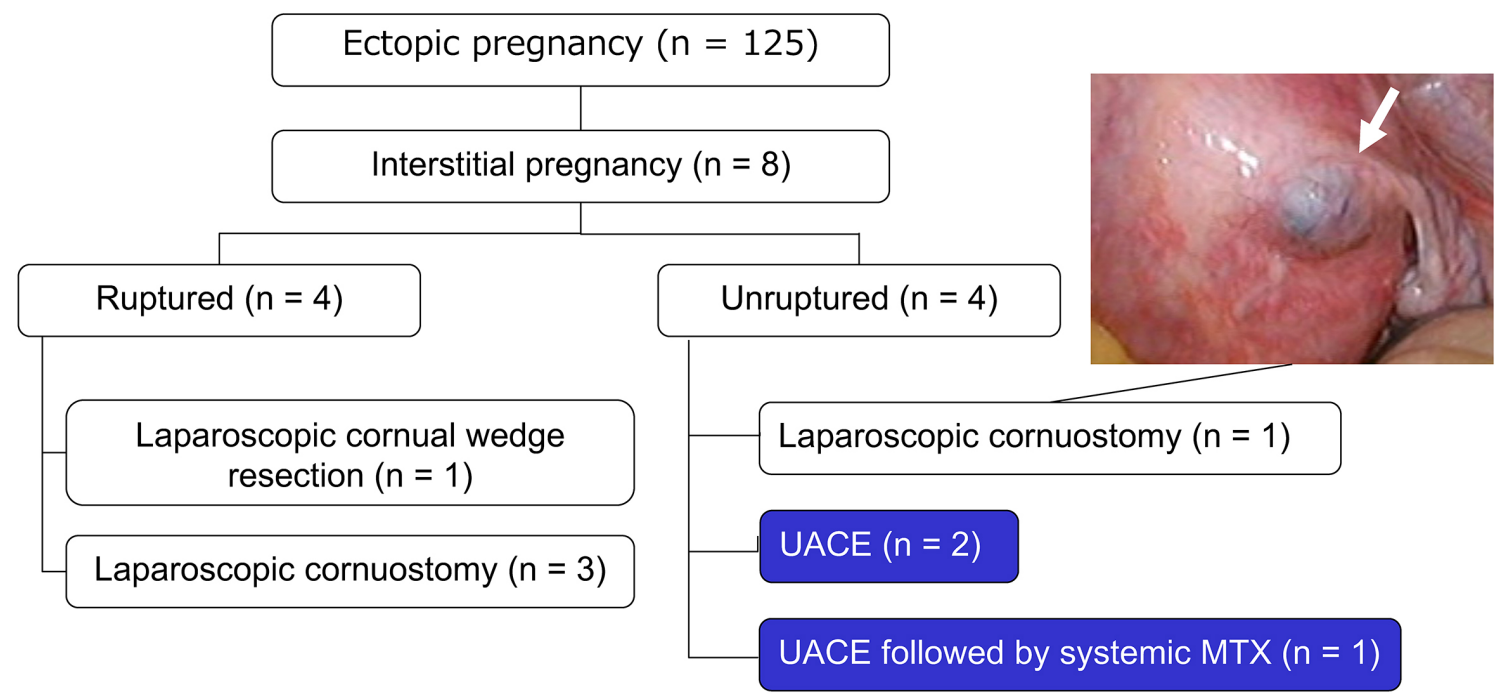

Fig. 1. Flow diagram of the classification of ectopic pregnancies experienced from 2014 to 2018 . Of 125 ectopic pregnancies, unruptured interstitial pregnancy was diagnosed in the early first trimester of pregnancy in four cases. One case was managed by laparoscopic cornuostomy due to concerns about cornual rupture because of thinning of the myometrium (inset, arrow). Two cases were successfully managed solely by uterine artery chemoembolization (UACE), while another case required systemic methotrexate administration after UACE to achieve complete resolution.

four women in the early first trimester of gestation. In one case with thinning of myometrial tissue detected under laparoscopic vision, laparoscopic cornuostomy was performed because of concerns about rupture, and this case was excluded (Fig. 1). The other three cases were initially managed by UACE and were included in the current investigation. The clinical course of each case is described below.

\subsection{Case 1: Proximal interstitial pregnancy managed by UACE after MRI diagnosis}

A 30-year-old, gravida 3, para 2 woman (Fig. 2) was referred at 6 weeks and 2 days of gestation because her physician suspected an ectopic pregnancy after she presented with amenorrhea following spontaneous conception.

At the initial referral, the serum $\beta$-hCG level was 4047 IU/L (Fig. 2D). Coronal T2-weighted MRI (Fig. 2A) revealed a gestational mass with a diameter of $8 \mathrm{~mm}$ in the muscular layer of the right interstitium adjacent to the uninterrupted junctional zone. A fetal heartbeat (FHB) was not detected. 3D-CTA (Fig. 2B) revealed a prominent vascular mass (Fig. 2B, arrow) localized in the right interstitial portion with blood supplied from the right uterine artery and the anastomosing left uterine artery.

After MRI-based diagnosis of an unruptured proximal interstitial pregnancy of the right fallopian tube, UACE was performed for immediate devascularization of gestational products (Fig. 2C, arrow). The serum $\beta$-hCG level dropped immediately after UACE (Fig. 2D). Nineteen days after UACE, the serum $\beta$-hCG level decreased to a normal level, and the natural ovulatory cycle resumed without any complications. Subsequent gestation was not reported, with no plans to conceive another child.

\subsection{Case 2: Distal interstitial pregnancy managed by UACE after laparoscopic diagnosis followed by a successful pregnancy outcome}

A 30-year-old, gravida 2, para 0 woman (Fig. 3) was referred at 6 weeks and 5 days of gestation under suspicion of ectopic pregnancy after conception by ovulation induced with clomiphene citrate followed by hCG injection. At triage, the serum $\beta$-hCG value was $3723 \mathrm{IU} / \mathrm{L}$ (Fig. 3C).

A gestational sac with a positive FHB in the left adnexal region adjacent to the uterus (data, not shown) was detected upon ultrasonographic examination. Coronal T2weighted MRI revealed a gestational mass-like structure with a diameter of $8 \mathrm{~mm}$ (Fig. 3A, arrow) at the left lateral angle of the uterine corpus, which led to a radiological diagnosis of left isthmic pregnancy rather than interstitial pregnancy.

A single-port laparoscopic view revealed an enlarged left interstitial portion (Fig. 3B, arrow) medial to the uteroovarian ligament (Fig. 3B, arrowhead) protruding from the interstitial portion, which indicated that gestational products exist in the distal interstitial portion of the fallopian tube.

Since early rupture was deemed improbable, UACE was performed for devascularization of gestational products. One day after UACE, there was a decrease in the serum $\beta$-hCG level, and the FHB could not be detected. Thirty-one days after UACE, the serum $\beta$-hCG level 

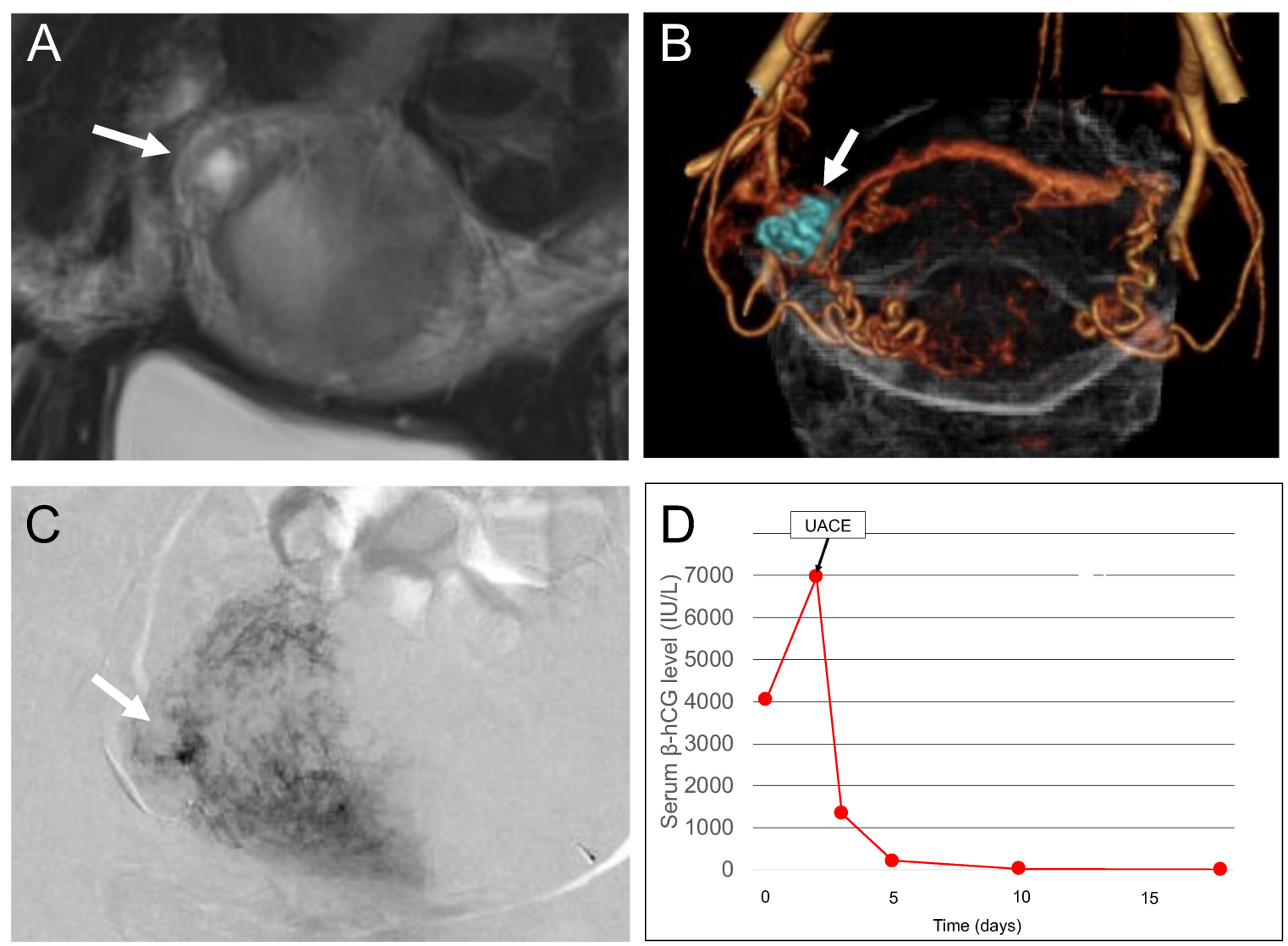

Fig. 2. Case 1: Proximal interstitial pregnancy managed by uterine artery chemoembolization (UACE) after diagnosis by magnetic resonance imaging (MRI). (A) Coronal T2-weighted MRI showing suspected gestational products with a diameter of $8 \mathrm{~mm}$ (arrow) in the proximal portion of the right interstitial uterine wall adjacent to the junctional zone. (B) Coronal three-dimensional computerized tomographic angiographic image in the arterial phase showing a prominent vascular mass (arrow) being fed by the right uterine artery and anastomosing left uterine artery in the right cornual region. (C) Digital subtraction angiography showing gestational products (arrow) supplied by the right uterine artery. (D) Changes in the serum $\beta$-hCG levels related to treatment. Nineteen days after UACE, the serum hCG value dropped to normal without any significant complications.

dropped below the normal range and no complications were reported. Three months after UACE, hysterosalpingography showed that patency of the left fallopian tube was restored (Fig. 3D, arrow).

Two years later, spontaneous conception occurred (side of ovulation unknown), followed by an uneventful vaginal birth at 38 weeks and 3 days of gestation resulting in a healthy, $2972 \mathrm{~g}$ female infant with Apgar scores of 9 and 10 at 1 and 5 minutes, respectively.

\subsection{Case 3: Distal interstitial pregnancy managed by UACE after a laparoscopic diagnosis followed by systemic MTX administration}

A 42-year-old, gravida 2, para 0 woman (Fig. 4) was referred at 6 weeks and 4 days of gestation under suspicion of right interstitial pregnancy with a positive FHB after conception by in vitro fertilization and embryo transfer.

At the initial examination, the serum $\beta$-hCG value was 6244 IU/L (Fig. 4D). Coronal T2-weighted MRI (Fig. 4A) revealed a gestational mass-like structure with a diameter of $7 \mathrm{~mm}$ (Fig. 4A, arrow) covered with a thin layer of myometrium at the right lateral angle of the uterine corpus.

Radiological diagnosis strongly indicated a distal interstitial pregnancy, although isthmic tubal pregnancy could not be completely excluded. Thereafter, single-port laparoscopy revealed a swollen right interstitial portion (Fig. 4B, arrow), which confirmed the diagnosis of right distal interstitial pregnancy, and pelvic adhesion due to peritoneal endometriosis was identified.

Since immediate rupture was deemed improbable, UACE was performed for devascularization of gestational products (Fig. 4C, arrow). One day after UACE, there was an immediate decrease in the serum $\beta$-hCG level, and the FHB could not be detected. However, due to re-elevation of the serum $\beta$-hCG value, systemic MTX administration was performed four times, resulting in the serum $\beta$-hCG level dropping below the normal range at 51 days after UACE without any complications. 

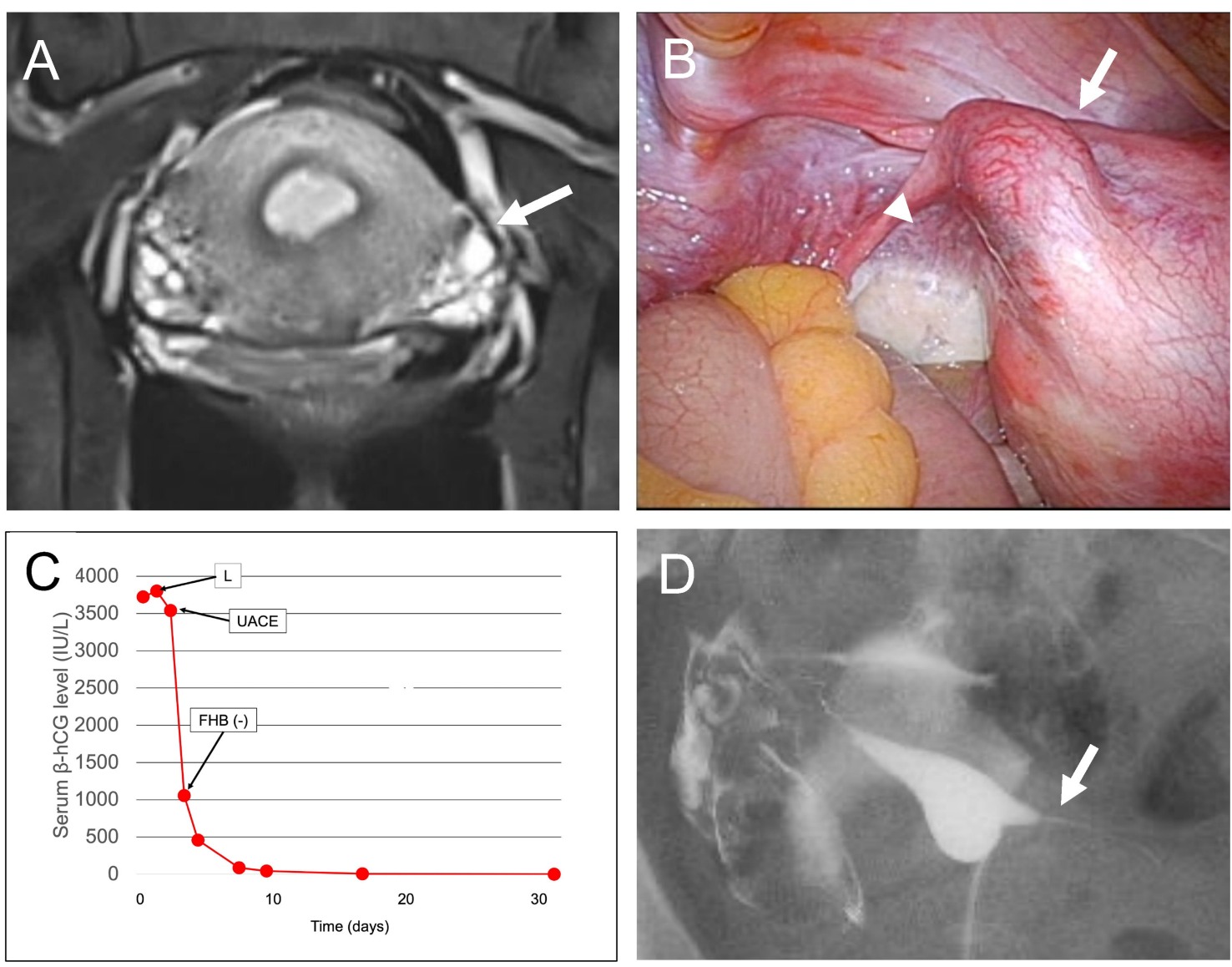

Fig. 3. Case 2: Distal interstitial pregnancy managed by uterine artery chemoembolization (UACE) after laparoscopic diagnosis, followed by a successful pregnancy outcome. (A) Coronal T2-weighted magnetic resonance imaging showing suspected gestational products with a diameter of $8 \mathrm{~mm}$ (arrow) in the left lateral angle of the uterine corpus, suggesting a radiological diagnosis of left isthmic pregnancy rather than interstitial pregnancy. (B) Single-port laparoscopic view showing an enlarged left interstitial portion (arrow) protruding from the uterine cornu median to uteroovarian ligament (arrowhead), indicating that gestational products exist in the distal portion of the interstitial fallopian tube. (C) Changes in the serum $\beta$-hCG levels related to treatment. After diagnosis by laparoscopy (L), uterine artery chemoembolization (UACE) was performed, followed by disappearance of the fetal heartbeat (FHB). Thirty-one days after UACE, the serum hCG value had dropped to normal without any significant complications. (D) Hysterosalpingography showed patency of the left fallopian tube (arrow) three months after UACE.

In this case, infertility therapy was resumed four months after UACE but was eventually terminated due to a complication of severe tubo-ovarian abscess at transvaginal oocyte retrieval.

\section{Discussion}

In replacing traditional laparotomy, early diagnosis of interstitial pregnancy before rupture makes various minimally invasive methods of treatment possible [3]. These medical management approaches include expectant management [3]; laparoscopic surgery [9] with reversible occlusion of uterine arteries and cornuostomy for advanced cases [18]; hysteroscopic management [6,10,14]; medical cytotoxic management [11] with mifepristone [12]; and endovascular therapy [8] with cytotoxic infusional chemotherapy [4].
Although such procedures, which can be performed independently or in combination [3], yield favorable outcomes with a satisfactory success rate, some adverse outcomes have been reported. In cases managed by laparoscopic surgery, interstitial rupture in subsequent gestation may lead to severe maternal and fetal morbidity [3], whereas in cases treated with expectant, medical, or endovascular therapy, incomplete resolution of the gestational tissue might require emergency surgical intervention due to interstitial rupture associated with significant hemorrhaging [19].

Based on our limited experiences with UACE for the management of unruptured interstitial pregnancies $[5$, 6], precise localization of gestational tissue by diagnostic imaging evaluations are needed to obtain successful outcomes. For diagnosis of interstitial pregnancy at early ges- 

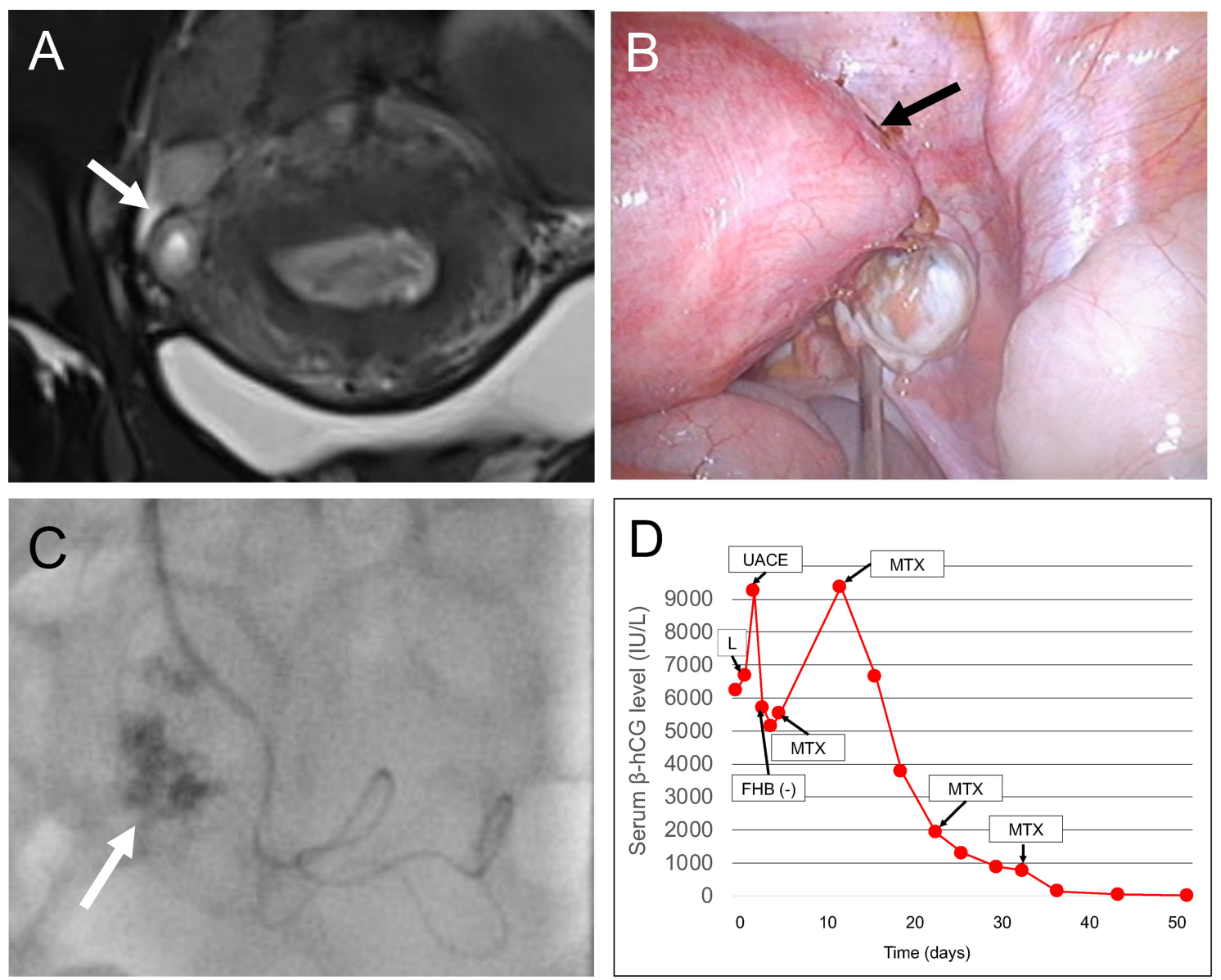

Fig. 4. Case 3: Distal interstitial pregnancy managed by uterine artery chemoembolization (UACE) after laparoscopic diagnosis, followed by systemic methotrexate administration. (A) Coronal T2-weighted magnetic resonance imaging showing suspected gestational products with a diameter of $7 \mathrm{~mm}$ (arrow) covered by a thin layer of myometrium in the right lateral angle of the uterine corpus. Radiological diagnosis indicated distal interstitial pregnancy, although isthmic tubal pregnancy could not be excluded. (B) A single-port laparoscopic view showing an enlarged right cornual portion indicated that gestational products exist in the distal portion of the interstitial fallopian tube. (C) Digital subtraction angiography showing gestational products (arrow) supplied by the right uterine artery. (D) Changes in serum $\beta$-hCG levels related to treatment. After a diagnosis by laparoscopy (L), UACE was performed, followed by disappearance of the fetal heartbeat (FHB). Although an immediate temporary decline in the serum $\beta$-hCG value was noted, re-elevation of the serum $\beta$-hCG value demanded an additional four rounds of cytotoxic therapy with methotrexate. Thereafter, the serum $\beta$-hCG level dropped below the normal range 51 days after UACE without any complications.

tational age, transvaginal ultrasonography with color-flow mapping is the first modality of choice [15]. Furthermore, three-dimensional ultrasound can precisely detect localization of the gestational sac to the interstitial portion of the fallopian tube [16].

However, when ultrasonography to differentiate interstitial pregnancy from an eccentrically implanted intrauterine pregnancy is inconclusive, it has been shown that MRI has a superior space-resolving ability compared to routine ultrasonography, as it can reveal a gestational sac-like structure within the myometrium $[5,6]$.

However, in the current study dealing with cases during the early first trimester, which therefore had small-size gestational products, the diagnostic ability of MRI was lim- ited when distal interstitial pregnancy was suspected, as in Cases 2 and 3, mainly because the uterine interstitial portion had a complex curved structure that could not be visualized well by conventional three-directional slices of MRI.

In such cases, exploratory laparoscopy is needed to achieve a final diagnosis of interstitial pregnancy, as it has been shown that many interstitial pregnancies are not practically diagnosed before surgery, with $56 \%$ to $71 \%$ of interstitial pregnancies mistaken for other types of ectopic pregnancy before surgery [3].

Spontaneous miscarriage is a common pregnancy outcome, whether the pregnancy is intrauterine or ectopic in location. Consequently, expectant management of distal tubal pregnancies is a treatment option when a spontaneous 
decline of serum $\beta$-hCG level is observed in asymptomatic women [3].

Similarly, expectant management of interstitial pregnancy has been successful in some cases [1]. However, complications include persistent pain requiring surgical excision even after successful management, catastrophic rupture and associated maternal morbidity and death, an unpredictable course to resolution even with declining serum $\beta$-hCG levels, and the need for prolonged hospitalization and resultant cost and disability [3].

In the current case series, retrospective speculation supposed that, especially in Case 2, expectant management could be an option because there was some degree of spontaneous decline in the serum $\beta$-hCG levels after laparoscopic diagnosis. However, this treatment option was not selected by the patient at that time after several management options including expectant management were offered.

Concerning the efficacy of UACE in resolving interstitial gestational products in three of the current cases, Cases 1 and 2 were successfully managed by UACE alone, whereas Case 3, re-elevation of the $\beta$-hCG level despite initial decline and potent feticidal effects presenting after UACE required additional MTX administration to achieve complete resolution.

Re-elevation of the serum $\beta$-hCG level after UACE in Case 3 indicates that endovascular embolization should only be recommended for occlusion of the bilateral uterine arteries, but not for anastomosing ovarian arteries because of the risk for ovarian failure due to ischemia or even necrosis of the embolized ovary.

Therefore, if interstitial gestation products receive more blood from the anastomosing ovarian artery than uterine arteries, complete resolution might not be achieved solely by UACE. Consequently, in such cases, additional MTX administration should be considered along with careful monitoring of interstitial rupture after poor decline of the serum $\beta$-hCG level.

Concerning uterine integrity with a focus on preserving future fertility after UACE, in Case 2, in which the patient reported a wish to retain fertility, UACE was successful, and hysterosalpingography after resolution of the gestation products showed restored patency of the affected fallopian tube followed by a successful pregnancy outcome after spontaneous conception, although the laterality of ovulation resulting in conception was not determined. More cases on this issue are required to validate the current findings so that reproductive and pregnancy outcomes in women undergoing different treatment approaches for interstitial pregnancy can be investigated, as different outcomes have been demonstrated for tubal ectopic pregnancies for which different management approaches were used [1].

At present time, the risk of recurrent interstitial pregnancy in subsequent gestation remains uncertain in cases with unruptured interstitial pregnancy treated with UACE due to the limited number of cases that were evaluated in the current study. However, important issues were raised by this study, which should be validated by an accumulation of future cases.

In the current cases, precise diagnosis in the early first trimester (all within six weeks of gestation) allowed unruptured interstitial pregnancy to be treated by UACE with or without subsequent systemic MTX administration; however, whether or not more advanced gestational cases with larger gestational products can be managed by the current procedure is unclear.

Based on our limited experience, laparoscopic surgery with or without endovascular therapy, as shown in our previous report [17], seems more appropriate for such cases than UACE due to the increased risk of a catastrophic outcome caused by interstitial rupture during conservative management [19].

Because several diagnostic and therapeutic modalities in addition to routine ultrasonography were used for management of these cases, the extra medical expenses present another concern regarding the studied treatment option. Further development of the modality, including the increased role of three-dimensional ultrasound, with an improvement of diagnostic ability are needed to overcome the challenges that we have encountered in our department.

\section{Conclusions}

The findings from this small case series suggest that UACE is a feasible, minimally invasive option for treating unruptured interstitial pregnancy diagnosed in the early first trimester in order to preserve the uterine structure and function, especially in women who wish to remain fertile.

\section{Author contributions}

AT and WK contributed to the conception of the study. AT, SI, ST, and WK contributed significantly to data analysis. AT wrote the manuscript. SI, ST, and WK helped in data collection. AT performed the analysis with constructive discussions. All authors contributed to editorial changes in the manuscript. All authors read and approved the final manuscript.

\section{Ethics approval and consent to participate}

All subjects gave their informed consent for inclusion before participation in the study. The study was conducted in accordance with the Declaration of Helsinki, and the protocol was approved by the Ethics Committee of Gifu Prefectural Tajimi Hospital (IRB number: 2020-OG-3).

\section{Acknowledgment}

We would like to thank Enago (https://www.enago.jp) for English language editing. 


\section{Funding}

This research received no external funding.

\section{Conflict of interest}

The authors declare no competing interests. AT is the Editorial board member of this journal, given his role as Editorial board member, AT had no involvement in the peerreview of this article and has no access to information regarding its peer-review.

\section{References}

[1] Baggio $\mathrm{S}$, Garzon $\mathrm{S}$, Russo A, Ianniciello CQ, Santi L, Laganà AS, et al. Fertility and reproductive outcome after tubal ectopic pregnancy: comparison among methotrexate, surgery and expectant management. Archives of Gynecology and Obstetrics. 2021; 303: 259-268.

[2] Garzon S, Raffaelli R, Montin U, Ghezzi F. Primary hepatic pregnancy: report of a case treated with laparoscopic approach and review of the literature. Fertility and Sterility. 2018; 110: 925-931.

[3] Moawad NS, Mahajan ST, Moniz MH, Taylor SE, Hurd WW. Current diagnosis and treatment of interstitial pregnancy. American Journal of Obstetrics and Gynecology. 2010; 202: 15-29.

[4] Gao J, Li X, Chen J, Gong W, Yue K, Wu Z. Uterine artery embolization combined with local infusion of methotrexate and 5- fluorouracil in treating ectopic pregnancy: a CONSORTcompliant article. Medicine. 2018; 97: e9722.

[5] Takeda A, Koike W, Hayashi S, Imoto S, Nakamura H. C. Magnetic resonance imaging for diagnosis and management of unruptured interstitial pregnancy. Journal of Obstetrics and Gynaecology Research. 2015; 41: 1384-1393.

[6] Takeda A, Koike W, Hayashi S, Imoto S, Nakamura H. Magnetic Resonance Imaging and 3-dimensional Computed Tomographic Angiography for Conservative Management of Proximal Interstitial Pregnancy by Hysteroscopic Resection after Transcatheter Arterial Chemoembolization. Journal of Minimally Invasive Gynecology. 2015; 22: 658-662.

[7] Hafner T, Aslam N, Ross JA, Zosmer N, Jurkovic D. The effectiveness of non-surgical management of early interstitial pregnancy: a report of ten cases and review of the literature. Ultrasound in Obstetrics and Gynecology. 1999; 13: 131-136.

[8] Deruelle P, Lucot J, Lions C, Robert Y. Management of interstitial pregnancy using selective uterine artery embolization. Ob- stetrics and Gynecology. 2005; 106: 1165-1167.

[9] Zuo X, Shen A, Chen M. Successful management of unruptured interstitial pregnancy in 17 consecutive cases by using laparoscopic surgery. Australian and New Zealand Journal of Obstetrics and Gynaecology. 2012; 52: 387-390.

[10] Leggieri C, Guasina F, Casadio P, Arena A, Pilu G, Seracchioli R. Hysteroscopic Methotrexate Injection under Ultrasonographic Guidance for Interstitial Pregnancy. Journal of Minimally Invasive Gynecology. 2016; 23: 1195-1199.

[11] Kim MJ, Cha J, Bae HS, Kim MK, Kim M, Yun BS, et al. Therapeutic outcomes of methotrexate injection in unruptured interstitial pregnancy. Obstetrics \& Gynecology Science. 2017; 60: 571-578.

[12] Stabile G, Romano F, Buonomo F, Zinicola G, Ricci G. Conservative Treatment of Interstitial Ectopic Pregnancy with the Combination of Mifepristone and Methotrexate: our Experience and Review of the Literature. BioMed Research International. 2020; 2020: 1-7.

[13] Ben-David A, Meyer R, Mohr-Sasson A, Mashiach R. Nonsurgical Management of Interstitial Pregnancies: Feasibility and Predictors of Treatment Failure. Journal of Minimally Invasive Gynecology. 2020; 27: 625-632.

[14] Casadio P, Arena A, Verrelli L, Ambrosio M, Fabbri M, Giovannico $\mathrm{K}$, et al. Methotrexate injection for interstitial pregnancy: Hysteroscopic conservative mini-invasive approach. Facts, Views and Vision in ObGyn. 2021; 13: 73-76.

[15] Timor-Tritsch IE, Monteagudo A, Matera C, Veit CR. Sonographic evolution of cornual pregnancies treated without surgery. Obstetrics and Gynecology. 1992; 79: 1044-1049.

[16] Jurkovic D, Mavrelos D. Catch me if you scan: ultrasound diagnosis of ectopic pregnancy. Ultrasound in Obstetrics \& Gynecology. 2007; 30: 1-7.

[17] Takeda A, Imoto S, Mori M, Nakano T, Nakamura H. Early experience with isobaric laparoendoscopic single-site surgery using a wound retractor for the management of ectopic pregnancy. European Journal of Obstetrics \& Gynecology and Reproductive Biology. 2011; 154: 209-214.

[18] Garzon S, Laganà AS, Pomini P, Raffaelli R, Ghezzi F, Franchi $M$. Laparoscopic reversible occlusion of uterine arteries and cornuostomy for advanced interstitial pregnancy. Minimally Invasive Therapy \& Allied Technologies. 2019; 28: 359-362.

[19] Takeda A, Koyama K, Imoto S, Mori M, Sakai K, Nakamura $\mathrm{H}$. Successful management of interstitial pregnancy with fetal cardiac activity by laparoscopic-assisted cornual resection with preoperative transcatheter uterine artery embolization. Archives of Gynecology and Obstetrics. 2009; 280: 305-308. 